\title{
La importancia de investigar Mycobacterium bovis en muestras clínicas de procedencia humana
}

\author{
Julio César Martínez¹, Claudia Llerena², Yanely Angélica Valbuena² \\ ${ }^{1}$ Centro de Investigación y Atención en Salud del Magdalena Medio, CISMAG, Barrancabermeja, \\ Colombia \\ ${ }^{2}$ Grupo de Micobacterias, Dirección de Redes en Salud Pública, Instituto Nacional de Salud, \\ Bogotá, D.C., Colombia
}

Introducción. La tuberculosis es una enfermedad infectocontagiosa que continúa siendo un problema mundial de salud pública. Es la principal causa de mortalidad en personas con HIV. Objetivo. Identificar la presencia de Mycobacterium bovis como agente etiológico de tuberculosis humana en muestras de esputo con baciloscopia positiva, mediante la prueba Genotype MTBC ${ }^{\mathrm{TM}}$.

Materiales y métodos. Se llevó a cabo un estudio descriptivo de 88 muestras de esputo remitidas al Grupo de Micobacterias del Instituto Nacional de Salud entre enero y noviembre de 2015. Se hizo el análisis microbiológico convencional y se empleó la prueba molecular de Genotype MTBC ${ }^{\text {TM }}$ para diferenciar las especies del complejo $M$. tuberculosis.

Resultados. Sesenta y dos casos (70,5 \%) correspondían a pacientes de sexo masculino; los grupos más afectados fueron el de 24 a 34 años, el de residentes en las cabeceras municipales, y el de afiliados al régimen subsidiado. En el $50 \%$ (44) de las muestras con resultados en la prueba de identificación de la especie, se detectó el complejo $M$. tuberculosis.

Conclusiones. La mayor carga de la enfermedad se registró en la población masculina y en edad productiva. La prueba de identificación para especies del complejo, solo demostró la presencia de $M$. tuberculosis. Sin embargo, con estos datos no es posible descartar $M$. bovis en humanos con tuberculosis en Colombia. La identificación diferencial de la especie debería implementarse de forma rutinaria en los casos de tuberculosis en los grupos de riesgo y en las zonas donde se conoce la circulación de esta micobacteria en bovinos.

Palabras clave: Mycobacterium bovis; Mycobacterium tuberculosis; tuberculosis; zoonosis; salud pública; Colombia.

Recibido: $15 / 02 / 18$

Aceptado: 04/09/18

Publicado: 05/09/18

Citación:

Martínez JC, Llerena C, Valbuena YA. La importancia de investigar Mycobacterium bovis en muestras clínicas de procedencia humana. Biomédica. 2019;39(Supl.1):117-24

https://doi.org/10.7705/biomedica.v39i2.4358

\section{Correspondencia:}

Julio César Martínez, Carrera 24S N 43-63,

Barrancabermeja, Colombia

Teléfono: (577) 6124956

jucer1023@hotmail.com

Contribución de los autores:

Julio Cesar Martínez y Claudia Llerena:

consolidación y análisis de la información

Yanely Angélica Valbuena: análisis microbiológico y

molecular de las muestras

Todos los autores participaron en la escritura del artículo.

Financiación:

Instituto Nacional de Salud de Colombia

Conflicto de intereses:

Los autores declaran no tener conflicto de intereses en relación con la preparación y la publicación de este trabajo.

\section{Importance of investigating Mycobacterium bovis in clinical samples of human origin}

Introduction: Tuberculosis is an infectious disease that still represents a major public health problem worldwide. It is one of the main causes of mortality in people with HIV. Objective: To identify the presence of $M$. bovis as an etiological agent of human tuberculosis in sputum smear positive samples using the test Genotype MTBCTM. Materials and methods: We conducted a descriptive study, 88 sputum samples were submitted to the Grupo de Micobacterias of the Instituto Nacional de Salud between January and November, 2015. We used the conventional microbiological analysis and the molecular test Genotype MTBC ${ }^{\mathrm{TM}}$ to identify the $M$. tuberculosis complex species.

Results: Sixty two (70.5\%) were males; the most affected groups were those between 24 and 34 years old, those residing in the municipal seats and those affiliated to the subsidized health plans. In $50.0 \%$ (44) of the samples with a result in the species identification test, we detected $M$. tuberculosis.

Conclusion: The highest burden of the disease was recorded among the male population in productive ages. The identification test for species of the complex showed all were $M$. tuberculosis. However, it is not possible to discard the presence of $M$. bovis in humans in Colombia. The differential identification of species should be done in risk groups and in areas where the circulation of this mycobacterium in cattle is known.

Keywords: Mycobacterium bovis; Mycobacterium tuberculosis; tuberculosis; zoonosis; public health; Colombia.

La tuberculosis humana es una enfermedad infectocontagiosa de distribución mundial, causada principalmente por Mycobacterium tuberculosis (1). Aunque este es el agente etiológico más frecuente, también puede ser causada por $M$. bovis, principal agente etiológico de la tuberculosis bovina (2). 
La Organización Mundial de la Salud (OMS) estimó que 10,4 millones de personas enfermarían de tuberculosis en el mundo en el 2015. En ese mismo año, se notificaron 6,1 millones de casos, alrededor de 4,3 millones no se diagnosticaron y 1,4 millones de muertes se atribuyeron a la enfermedad (3). Estas cifras ubican la tuberculosis como una de las mayores causas de morbilidad y mortalidad de origen infeccioso a nivel mundial, y la situación es más alarmante en los países de bajos y medianos ingresos, en los cuales ocurren de 95 a $98 \%$ de los casos, principalmente en las personas infectadas por el virus de la inmunodeficiencia humana (HIV) (4).

La situación en Colombia no está lejos de esta problemática mundial, pues en el 2017 se notificaron 14.187 casos de tuberculosis incluidas todas sus formas, con una incidencia de 26 casos por 100.000 habitantes. Además, se registraron 1.083 muertes debidas a la enfermedad, con una tasa de mortalidad de 2,25 muertes por 100.000 habitantes (5).

En la actualidad no se conoce la magnitud real de la morbilidad atribuida a $M$. bovis; sin embargo, se estima que el $3 \%$ de los casos de tuberculosis ocurridos en el mundo se deben a esta micobacteria. La transmisión zoonótica ocurre principalmente por el consumo de productos lácteos o de sus derivados sin pasteurizar, y por el contacto con animales y humanos infectados (6-8).

En la región de las Américas, la tuberculosis por $M$. bovis en humanos se ha documentado en varios países. Argentina tiene una prevalencia estimada entre 0,4 y $6,0 \%$, superada por algunas zonas endémicas de México, con cifras de hasta 13,8\%. En otras áreas, como en la frontera sur de los Estados Unidos, también se ha documentado que cerca del $70 \%$ de los casos de tuberculosis son causados por $M$. bovis, con la mayor proporción en personas de origen mexicano (9-12). Entre 1996 y 2006, se registraron tres casos en Brasil y, entre 1998 y 2005, dos casos en Ecuador y tres en Uruguay, lo que indica que en los países de la región la prevalencia es baja, aunque debe anotarse que hay subregistro de casos $(13,14)$.

Los signos y síntomas clínicos de la tuberculosis producida por M. bovis no se diferencian de los de la enfermedad causada por $M$. tuberculosis, lo que dificulta su diagnóstico y exige que su diferenciación se haga mediante técnicas moleculares $(15,16)$. Esta diferenciación de especies es importante para establecer el esquema de tratamiento, debido a que las cepas de $M$. bovis tienen resistencia natural a la pirazinamida $(17,18)$.

Varias instituciones de la red de laboratorios de tuberculosis de Colombia, utilizan las pruebas moleculares en las muestras directas. En el caso de los laboratorios de salud pública, se recurre a estos métodos a partir de los cultivos positivos. Son pocos los casos de $M$. bovis identificados en humanos, debido a que la diferenciación de especies del complejo $M$. tuberculosis no se hace de forma rutinaria y los métodos disponibles no tienen este alcance.

El objetivo de este trabajo fue identificar las especies del complejo $M$. tuberculosis en muestras de esputo con baciloscopia positiva, recibidas en el Grupo de Micobacterias del Instituto Nacional de Salud entre enero y noviembre de 2015.

\section{Materiales y métodos}

Se hizo un estudio descriptivo, en el cual se incluyeron 88 muestras de esputo con baciloscopia positiva provenientes de personas con antecedentes de tratamiento contra la tuberculosis en el marco de las acciones de 
vigilancia, entre enero y noviembre de 2015. Dichas muestras se priorizaron para la prueba molecular de identificación de la especie. Las muestras habían sido remitidas por algunos de los laboratorios de salud pública del país que no cuentan con estas metodologías.

Se hizo la baciloscopia de las muestras empleando la coloración de Ziehl-Neelsen; se descontaminaron con el método de $\mathrm{NaOH}-\mathrm{N}$-acetil-Lcisteína, y el ADN se extrajo con el estuche GenoLyse DNA Extraction ${ }^{\mathrm{TM}}$ $(19,20)$. La prueba molecular empleada fue la Genotype MTBC ${ }^{\text {TM }}$, la cual permite diferenciar las especies del complejo $M$. tuberculosis, siguiendo las instrucciones del fabricante (21).

Los casos se graficaron en un mapa con la herramienta ArcGIS ${ }^{\text {TM }}$ en su versión de prueba, para establecer la distribución geográfica según el origen de los pacientes.

Con la información registrada en el formato único de vigilancia de las micobacterias, se analizaron las siguientes características sociodemográficas: entidad territorial de procedencia de la muestra en términos de frecuencias absolutas y relativas; sexo; lugar de residencia; afiliación al Sistema General de Seguridad Social en Salud; pertenencia étnica; edad y resultado de la prueba de identificación de la especie. Esta información se incluyó en una base de datos de Excel ${ }^{\mathrm{TM}}$ para su análisis.

\section{Consideraciones éticas}

El estudio se rigió por los principios metodológicos requeridos para salvaguardar el interés de la ciencia y el respeto a los derechos de las personas. En los procedimientos no hubo ninguna intervención en humanos y no se violó el derecho a la privacidad, ya que los resultados se presentaron de manera anónima.

\section{Resultados}

Cuarenta y siete de las 88 (53,4 \%) muestras analizadas procedía de los departamentos en los que se ha comprobado la circulación de $M$. bovis en bovinos (22): Norte de Santander, 28 (31,8\%); Casanare, 6 (6,8\%); Boyacá, 5 (5,7 \%); Nariño, 4 (4,5\%), y Cesar, Magdalena, La Guajira y Cundinamarca, una $(1,1 \%)$ cada uno. Las $41(46,6 \%)$ restantes procedían de entes territoriales en los cuales no se ha confirmado la presencia de tuberculosis bovina (22): Tolima, 14 (15,9\%); Risaralda, 6 (6,8\%); Huila, 5 (5,7\%); Caldas, 4 (4,5\%); Arauca, 3 (3,4 \%) y Bolívar, 3 (3,4 \%), seguidos por Santander con $2(2,3 \%)$, Sucre con 2 (2,3\%), y Quindío y Bogotá, cada uno con 1 (1,1\%) (figura 1).

El análisis de las características sociodemográficas indicó que el $70,5 \%$ de los casos se registró en hombres y $71(80,7 \%)$ residía en la cabecera municipal. En cuanto al tipo de afiliación al Sistema General de Seguridad Social en Salud, $57(64,8 \%)$ pertenecía al régimen subsidiado, 12 (13,6\%) se encontraba sin afiliación y el porcentaje restante pertenecía al régimen contributivo. En cuanto a la pertenencia étnica, 79 (89,8\%) se clasificó en la categoría de otros grupos étnicos. Al categorizar la edad por grupos, la mayor proporción (19,3\%) $(n=17)$ de casos se concentró en los adultos jóvenes (de 25 a 34 años), seguido por el grupo de adulto medio (de 45 a 54 años), con 18,2\% ( $n=16)$, y el de adultos mayores (de 65 y más años), con 15,9\% ( $n=14)$ (cuadro 1).

En el $50 \%(n=44)$ de las muestras se identificó la especie M. tuberculosis, en tanto que en las demás no se obtuvo amplificación del ADN. 


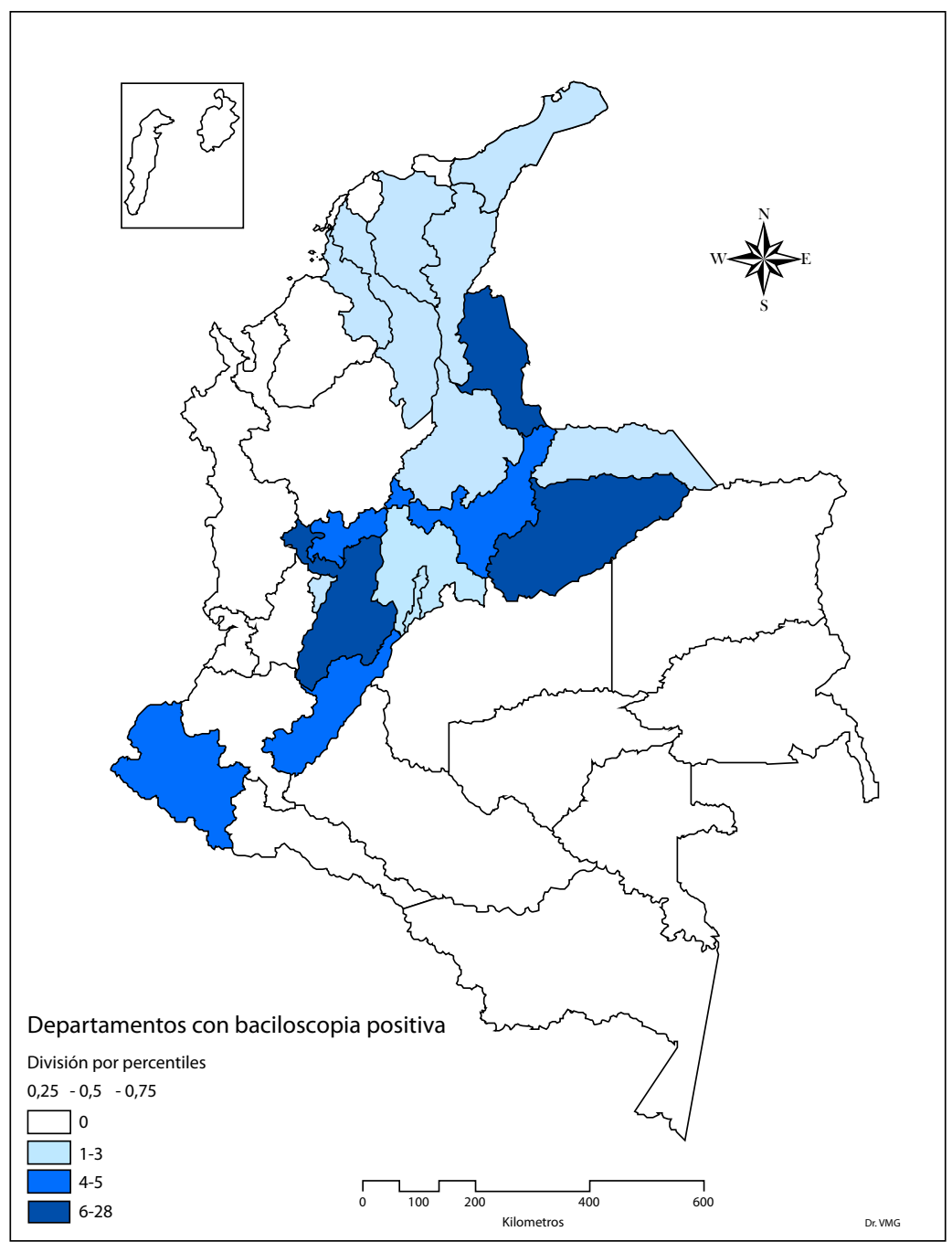

Fuente: Laboratorio Nacional de Referencia de Micobacterias, Instituto Nacional de Salud, Colombia, 2015

Figura 1. Distribución geográfica de los casos con baciloscopia positiva y antecedentes de tratamiento de tuberculosis, Colombia, 2015

\section{Discusión}

Los casos analizados provenían de entidades territoriales que deben enviar al Laboratorio Nacional de Referencia las muestras de pacientes previamente tratados, como parte del esquema de vigilancia de la resistencia. No se recibieron muestras de los departamentos que habitualmente notifican un número importante de casos de tuberculosis, como Antioquia, Atlántico y Valle del Cauca (5), debido a que sus respectivas redes de laboratorios cuentan con pruebas rápidas y no requieren el apoyo del Laboratorio Nacional de Referencia.

Según la información del Instituto Colombiano Agropecuario, en Colombia la prevalencia de tuberculosis bovina es inferior al 1,0\% y, en general, en el país se ha evidenciado un descenso en el número de casos en los últimos años, con excepción de algunas áreas ubicadas en los departamentos de Boyacá y Cundinamarca, donde se ha demostrado la persistencia de la circulación de $M$. bovis (23), lo cual representa un riesgo latente de zoonosis, no solo para los manipuladores directos de las fincas en saneamiento, sino también, para los manipuladores de la cadena productiva y para los consumidores finales. 
Cuadro 1. Comportamiento de las características sociodemográficas de las personas con baciloscopia positiva y antecedentes de tratamiento de tuberculosis analizados para la identificación de M. bovis, Colombia, 2015

\begin{tabular}{|c|c|c|}
\hline Variable & $\mathbf{n}$ & $\%$ \\
\hline \multicolumn{3}{|l|}{ Sexo } \\
\hline Hombre & 62 & 70,5 \\
\hline Mujer & 26 & 29,5 \\
\hline Total & 88 & 100 \\
\hline \multicolumn{3}{|l|}{ Área de procedencia } \\
\hline Cabecera municipal & 71 & 80,7 \\
\hline Rural disperso & 13 & 14,8 \\
\hline Centro poblado & 4 & 4,5 \\
\hline Total & 88 & 100 \\
\hline \multicolumn{3}{|l|}{$\begin{array}{l}\text { Régimen de afiliación al Sistema General de } \\
\text { Seguridad Social en Salud }\end{array}$} \\
\hline Subsidiado & 57 & 64,8 \\
\hline No asegurado & 12 & 13,6 \\
\hline Sin información & 11 & 12,5 \\
\hline Contributivo & 7 & 8,0 \\
\hline Excepción & 1 & 1,1 \\
\hline Total & 88 & 100 \\
\hline \multicolumn{3}{|l|}{ Pertenencia étnica } \\
\hline Otros & 79 & 89,9 \\
\hline Indígena & 4 & 4,5 \\
\hline Afrocolombiano, afrodescendiente, mulato & 4 & 4,5 \\
\hline ROM, gitano & 1 & 1,0 \\
\hline Total & 88 & 100 \\
\hline \multicolumn{3}{|l|}{ Grupos de edad (años) } \\
\hline$<15$ & 0 & 0,0 \\
\hline 15 a 24 & 15 & 17,0 \\
\hline 25 a 34 & 17 & 19,3 \\
\hline 35 a 44 & 12 & 13,6 \\
\hline 45 a 54 & 16 & 18,2 \\
\hline 55 a 64 & 14 & 15,9 \\
\hline$\geq 65$ & 14 & 15,9 \\
\hline Total & 88 & 100 \\
\hline
\end{tabular}

Fuente: Laboratorio Nacional de Referencia de Micobacterias, Instituto Nacional de Salud, Colombia, 2015

En cuanto al comportamiento según sexo y grupos de edad, los datos obtenidos fueron similares a la información oficial sobre la caracterización de la tuberculosis a nivel nacional, pues la mayor carga de la enfermedad se ha observado en la población masculina, principalmente en adultos jóvenes en edad productiva (5). El 80,7\% ( $n=71)$ de los casos estudiados provenía de zonas urbanas, lo que limita la posibilidad de identificar M. bovis, pues su presencia se relaciona con el contacto con animales enfermos y la manipulación de productos cárnicos o lácteos sin pasteurizar, actividades netamente agrícolas.

En cuanto al régimen de afiliación al sistema de salud, la mayoría de los casos $(64,8 \% ; n=57)$ pertenecía al subsidiado y un porcentaje importante $(13,6 \% ; n=12)$ no estaba asegurado, lo que demuestra que la mayor carga de la enfermedad se presenta en la población pobre que no cuenta con seguridad social, condición que agrava la situación del paciente, pues el tratamiento depende del suministro de medicamentos por parte del programa nacional de tuberculosis que, afortunadamente, ha tenido buenos resultados en el país en cuanto a su sostenibilidad y la permanencia de los pacientes. Sin embargo, un paciente que no esté vinculado al régimen de salud supone la misma condición para sus contactos, lo que favorece la diseminación del 
bacilo, pues su captación, diagnóstico y tratamiento se hacen de manera más tardía, en comparación con los pacientes del régimen contributivo (24).

El Instituto Nacional de Salud ha hecho dos encuestas nacionales de vigilancia de la resistencia a los fármacos antituberculosos en cerca de 5.000 personas con tuberculosis pulmonar y baciloscopia positiva provenientes de todo el país. Las muestras de esputo se sembraron en medios Ogawa-Kudoh y Stonebrink modificado por Giraldo, este último con el fin de identificar si había circulación de $M$. bovis. No hubo ningún cultivo en el que se detectara esta especie, lo cual podría deberse a que la técnica de cultivo utilizada no es lo suficientemente sensible para detectar pocos bacilos, algo que es característico de esta especie $(25,26)$.

En el 2012, el Grupo de Micobacterias de la Subdirección de Investigación e Innovación del Instituto Nacional de Salud detectó un caso de tuberculosis causado por $M$. bovis en un individuo de 58 años de edad, información que generó nueva evidencia sobre la presencia de esta micobacteria en humanos (27).

En el 2016, Leal, et al., analizaron 164 muestras de esputo de trabajadores de fincas con circulación de $M$. bovis en ganado y no encontraron casos de tuberculosis debidos a este agente etiológico; los datos que obtuvieron son similares a los de este estudio, a pesar de que los grupos de población analizados eran diferentes en términos de la exposición reconocida (28).

En la región de las Américas se estima una prevalencia de tuberculosis por M. bovis de menos de 0,05 \% (13). Según la OMS, en el 2013 se presentaron 231.330 casos, aunque esta cifra está subestimada debido a que en muchos países no se cuenta con técnicas de diferenciación de las especies del complejo M. tuberculosis, las cuales deben ser de comprobada sensibilidad y con suficiente poder discriminatorio, como la técnica de hibridación inversa de spoligotyping. En varios estudios de genómica comparativa, se ha evidenciado que las regiones de diferenciación presentes en $M$. bovis muestran una pérdida de material genético con relación a $M$. tuberculosis, lo que hace que su estudio permita conocer más sobre las relaciones filogenéticas de las especies que conforman el complejo $(11,29,30)$.

Algunas de las limitaciones de este trabajo se relacionan con el número de muestras analizadas, ya que no es representativo del total de casos de tuberculosis notificados en ese periodo a nivel nacional. Además, la técnica empleada solo permitió identificar la especie en el $50 \%(n=44)$ de las muestras, debido a que la prueba GenotypeMTBC ${ }^{\mathrm{TM}}$ no está recomendada para muestras con baciloscopia positiva. Por esta razón, no se descarta la posibilidad de que se presente esta zoonosis en los departamentos de Colombia analizados.

Se concluyó que la especie identificada en las muestras como causante de enfermedad fue $M$. tuberculosis. Para evidenciar la presencia de $M$. bovis como causante de tuberculosis en humanos, se requiere que las muestras provengan de personas con factores de riesgo, como manipuladores de ganado y productos cárnicos, médicos veterinarios, zootecnistas e individuos residentes en zonas con circulación de esta micobacteria. Tales muestras deben sembrarse en medios de cultivo que permitan el crecimiento de esta especie y, en caso de resultar positivas, deben evaluarse con técnicas que diferencien las especies del complejo. 
En Colombia deben desarrollarse más estudios piloto encaminados a implementar nuevas estrategias de vigilancia para mejorar el diagnóstico por laboratorio y analizar variables como tipo de tuberculosis (pulmonar, extrapulmonar), lugar de residencia del individuo, ocupación, tipo de afiliación al Sistema General de Seguridad Social en Salud y estatus de HIV, entre otros aspectos analizados en estudios previos (28), como el tiempo de exposición del trabajador (permanencia de más de un año en la finca), el nivel de escolaridad, los conocimientos sobre la zoonosis y la cercanía con otros huéspedes, todo ello articulado con el Instituto Colombiano Agropecuario, para establecer una línea de base de la transmisión de $M$. bovis en humanos. Solo así se podrían diseñar políticas de salud pública para combatir este problema.

\section{Agradecimientos}

A los profesionales y a los laboratorios de salud pública que remitieron muestras al Laboratorio Nacional de Referencia del Instituto Nacional de Salud.

\section{Referencias}

1. Tortoli E. Microbiological features and clinical relevance of new species of the genus Mycobacterium. Clin Microbiol Rev. 2014;27:727-52. https://doi:10.1128/CMR.00035-14

2. Ramos DF, Silva AB, Fagundes MQ, von Groll A, Almeida PE, Dellagostin OA. Molecular typing of Mycobacterium bovis isolated in the south of Brazil. Braz J Microbiol. 2014;45:65760. https://doi.org/10.1590/S1517-83822014000200039

3. World Health Organization. Global Tuberculosis Report, 2016. Fecha de consulta: 5 de diciembre de 2017. Disponible en:

http://apps.who.int/medicinedocs/documents/s23098en/s23098en.pdf

4. Zumla A, Granje J. Doing something about tuberculosis. BMJ. 1999;318:956-8. https://doi.org/10.1136/bmj.318.7189.956

5. Instituto Nacional de Salud. Informe del evento preliminar de tuberculosis, hasta el periodo epidemiológico XIII, Colombia, 2017. Fecha de consulta: 18 de abril de 2018. Disponible en: http://www.ins.gov.co/buscador-eventos/Informesdeevento/TUBERCULOSIS\%20PE\%20 XIII\%202017.pdf

6. Cosivi O, Grange JM, Daborn CJ, Raviglione MC, Fujikura T, Cousins D, et al. Zoonotic tuberculosis due to Mycobacterium bovis in developing countries. Emerg Infect Dis. 1998;4:59-70. https://loi.org/10.3201/eid0401.980108

7. Torres P, Soberanis O, Martínez A, Chávez B, Barrios MT, Torres M, et al. Prevalence of latent and active tuberculosis among dairy farm workers exposed to cattle infected by Mycobacterium bovis. PLoS Negl Trop Dis. 2013;7:1-8. https://doi.org/10.1371/journal.pntd.0002177

8. Üller B, Durr S, Alonso S, Hattendorf J, Laisse C, Parsons S, et al. Zoonotic Mycobacterium bovis induced tuberculosis in humans. Emerg Infect Dis. 2013;19:899-908. https://doi.org/10.3201/eid1906.120543

9. Abalos P, Retamal P. Tuberculosis: ¿una zoonosis re-emergente? Rev Sci Tech Offlnt Epiz. 2004;23:583-94.

10. Pérez L, Navarro Y, García de Viedma D. Current knowledge and pending challenges in zoonosis caused by Mycobacterium bovis: A review. Res Vet Sci. 2014;97:S94-S100. https://doi.org/10.1016/j.rvsc.2013.11.008

11. Kantor IN, Ambroggib M, Poggib S, Morcilloc N, Da Silva MA, Osorio M, et al. Human Mycobacterium bovis infection in ten Latin American countries. Tuberculosis. 2008 88:35865. https://doi.org/10.1016/j.tube.2007.11.007

12. Pérez L, Milian F, Arriaga C, Romero C, Escartin M. Epidemiología molecular de la tuberculosis bovina y humana en una zona endémica de Querétaro, México. Salud Pública Mex. 2008;50:286-91.

13. Kantor IN, LoBue PA, Thoen CO. Human tuberculosis caused by Mycobacterium bovis in the United States, Latin America and the Caribbean. Int J Tuberc Lung. 2010;14:1369-73.

14. Rivas C, Greif G, Coitinho C, Araújo L, Laserra P, Robello C. Primeros casos de tuberculosis pulmonar por Mycobacterium bovis. Una zoonosis reemergente en Uruguay. Rev Med Urug. 2012;28:209-14. 
15. Park D, Qin H, Jain S, Preziosi M, Minuto J, Mathews C, et al. Tuberculosis due to Mycobacterium bovis patients coinfected with human inmunodeficiency virus. Clin Infect Dis. 2010;51:1343-6. https://doi.org/10.1086/657118

16. Hlavsa M, Moonan P, Cowan L, Navin T, Kammerer S, Morlock G, et al. Human tuberculosis due to Mycobacterium bovis in the United States, 1995-2005. Clin Infect Dis. 2008;47:16875. https://doi.org/10.1086/589240

17. Kamerbeek J, Schouls L, Kolk A, van Agterveld M, van Soolingen D, Kuijper S, et al. Simultaneous detection and strain differentiation of Mycobacterium tuberculosis for diagnosis and epidemiology. J Clin Microbiol 1997;35:907-14.

18. Aranaz A, Liébana E, Mateos A, Domínguez L, Vidal D, Domingo M, et al. Spacer oligonucleotide typing of Mycobacterium bovis strains from cattle and other animals: A tool for studying epidemiology of tuberculosis. J Clin Microbiol 1996;34:2734-40.

19. Organización Panamericana de la Salud. Manual para el diagnóstico bacteriológico de la tuberculosis. Normas y guía técnica. Cultivo. Fecha de consulta: 5 de diciembre de 2017 Disponible en: http://www.sld.cu/galerias/pdf/sitios/tuberculosis/tb-labs-cultivo[2].pdf

20. Organización Panamericana de la Salud. Manual para el diagnóstico bacteriológico de la tuberculosis. Normas y guía técnica. Baciloscopia. Fecha de consulta: 5 de diciembre de 2017. Disponible en: http://www.sld.cu/galerias/pdf/sitios/tuberculosis/tb-labs-baciloscopia[1].pdf

21. Hain Lifescience $\mathrm{GmbH}$. Insertotécnica Genotype MTBC ${ }^{\mathrm{TM}}$ para la diferenciación de especies del complejo M. tuberculosis. Nehren, Alemania: Hain Lifescience GmbH; 2012.

22. Federación Colombiana de Ganaderos. Tuberculosis bovina. Fecha de consulta: 20 de abril de 2018. Disponible en: http://www.fedegan.org.co/programas/tuberculosis-bovina

23. Instituto Colombiano Agropecuario. Boletín epidemiológico anual, Colombia sanidad animal, 2014. Fecha de consulta: 20 de abril de 2018. Disponible en: https://www.ica.gov.co/Areas/ Pecuaria/Servicios/Epidemiologia-Veterinaria/Bol/Epi/Boletines-Anuales.aspx

24. Ministerio de Salud y Protección Social. Programa Nacional de Prevención y Control de la Tuberculosis. Plan de monitoreo y evaluación. Fecha de consulta: 22 de diciembre de 2017. Disponible en: https://www.minsalud.gov.co/sites/rid/Lists/BibliotecaDigital/RIDE/VS/PP/ET/ plan-monitoreo-evaluacion-tuberculosis.pdf

25. León Cl, Sierra C, Naranjo N, Garzón MC, Guerrero MI. Segundo estudio nacional de resistencia primaria de $\mathrm{M}$. tuberculosis a las drogas antituberculosas en Colombia. Infectio. 2002;6:83.

26. Garzón MC, Angee DY, Llerena C, Orjuela D, Victoria J. Vigilancia de la resistencia de Mycobacterium tuberculosis a los fármacos antituberculosos, Colombia 2004-2005. Biomédica. 2008;28:319-26. https://doi.org/10.7705/biomedica.v28i3.71

27. Puerto G, Erazo L, Wintaco M, Castro C, Ribón W, Guerrero MI. Mycobacterium tuberculosis genotypes determined by spoligotyping to be circulating in Colombia between 1999 and 2012 and their possible associations with transmission and susceptibility to firstline drugs. PLoS One. 2015;10:e0124308. https://doi.org/10.1371/journal.pone.0124308

28. Leal AF, Castro CM, Wintaco LM, Villalobos R, Puerto GM. Tuberculosis por Mycobacterium bovis en trabajadores de fincas en saneamiento para tuberculosis bovina, de Antioquia, Boyacá y Cundinamarca. Rev Salud Pública. 2016;18:727-37. https://doi.org/10.15446/rsap.v18n5.51187

29. Kamerbeek J, Schouls L, Kolk A, van Agterveld M, van Soolingen D, Kuijper S, et al. Simultaneous detection and strain differentiation of Mycobacterium tuberculosis for diagnosis and epidemiology. J Clin Microbiol. 1997;35:907-14.

30. Arráiz N, Romay Z, Faría N, Mujica D. Identificación diferencial de aislados clínicos de Mycobacterium tuberculosis y Mycobacterium bovis por un ensayo de PCR múltiple. Rev Cient. 2006;16:622-8. 